\title{
Assessment of Likelihood Ratio for Four Contact Dermatitis Symptoms of Vinca Minor
}

\author{
Suman Bagchi ${ }^{1,2}$ Chaturbhuja Nayak ${ }^{1,3}$ Lex Rutten ${ }^{4}$ Munmun Koley ${ }^{5}$ Subhranil Saha ${ }^{6}$ \\ Mousumi Roy ${ }^{7}$ Sk Jahir Abbas ${ }^{8}$ Simantini Ghosh ${ }^{9}$ Suman Halder $^{10}$ Ghulam Nabi Wani $^{11}$ \\ Anaitulah Ahmad Mir ${ }^{11}$ Showket Ahmad Bhat ${ }^{11}$
}

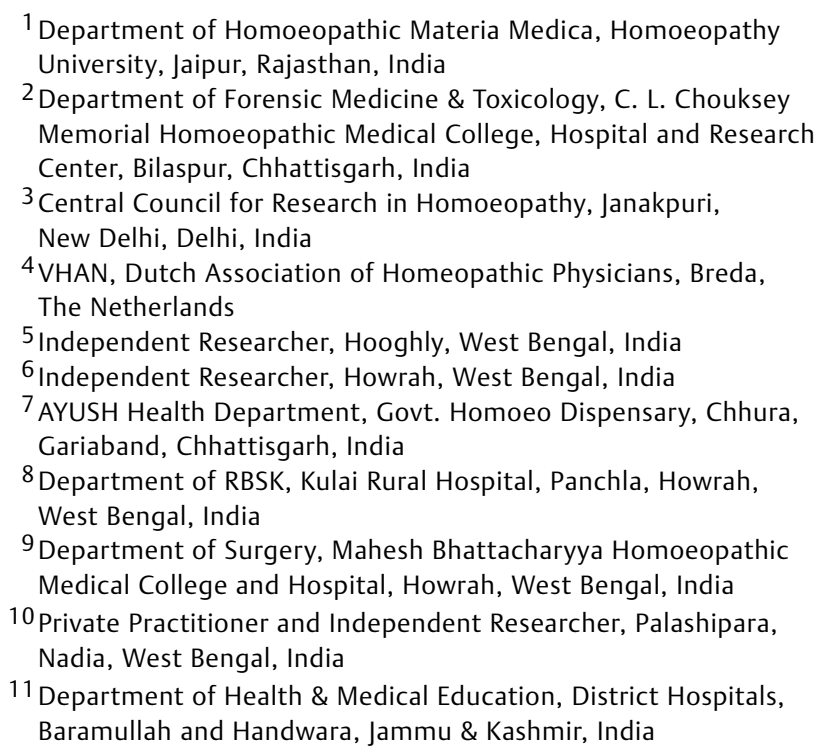

Address for correspondence Suman Bagchi, MD(Hom), Department of Forensic Medicine \& Toxicology, C. L. Chouksey Memorial Homoeopathic Medical College, Hospital and Research Center, Bilaspur, Chattisgarh, India (e-mail: drsbagchi@gmail.com).

\begin{abstract}
Keywords

- homeopathy

- Vinca minor

- contact dermatitis

- likelihood ratio

Background Contact dermatitis (CD) is a frequently occurring medical condition, for which Vinca minor (VM) is one of the recommended homeopathic medicines. However, the symptoms indicating this medicine have not yet been assessed systematically. Likelihood ratio (LR), based on Bayesian statistics, may yield better estimation of a medicine's indication than the existing method of entry of symptoms into materia medica and repertories.

Methods We investigated LRs of four CD symptoms of VM: (1) great sensitiveness of skin, with redness and soreness from slightest rubbing; (2) weeping eczema with foul, thick crusts; (3) itching amelioration in open air; and (4) CD of scalp. An observational, prospective, patient-outcome study was conducted in five different practice settings on 390 CD patients over 18 months using three outcomes-Glasgow Homeopathic Hospital Outcome Scale (GHHOS), Scoring Atopic Dermatitis (SCORAD), and Dermatology Life Quality Index (DLQI), assessed at baseline, after 3 and 6 months. The LR of each of the four symptoms was estimated as per the patient-rated outcomes on GHHOS.

Results Seventy-four VM and 316 non-VM cases were analyzed. Estimated LRs were as follows: symptom 1, 1.29 (95\% confidence interval [CI]: 0.65 to 2.60); symptom 2, 1.48 (95\% Cl: 0.80 to 2.74 ); symptom 3, 1.70 ( $95 \% \mathrm{Cl}$ : 0.94 to 3.07 ); symptom 4, 1.36 (95\%
\end{abstract}

received

March 6, 2018

accepted after revision

April 11, 2018

published online

May 21, 2018
Copyright $\odot 2018$ The Faculty of Homeopathy
DOI https://doi.org/ 10.1055/s-0038-1654713. ISSN 1475-4916. 
$\mathrm{Cl}: 0.74$ to 2.51$)$. There were statistically significant reductions in SCORAD and DLQI scores over 3 and 6 months.

Conclusion There was insufficient evidence to attribute any of the four assessed symptoms clearly to VM. Though non-significant, a high LR was observed for "itching amelioration in open air" (symptom 3). Symptoms in the homeopathic materia medica for VM are perhaps over-represented. More research of this nature is warranted.

\section{Introduction}

Contact dermatitis (CD) is a pathological entity for which occupational exposure can be presented to be a primary cause or contributory element. $C D$ is prevalent in the population and the long-term prognosis for individuals is poor unless harmful exposures are addressed. Not only do they continue to have disease and their overall quality of life (QoL) may be impacted, but also they may have significant work disruption. The two most common types of $\mathrm{CD}$ are allergic and irritant. ${ }^{1-3}$ In most cases, both types present as eczematous lesions on uncovered parts of the body, particularly the hands. Eczematous lesions can have acute (erythema, edema, vesicles, exudation), subacute (exudation, crusts), and chronic (xerosis, scaling, keratosis, lichenization, fissures) evolution. ${ }^{4}$ The impact of $\mathrm{CD}$ is often undervalued because the course of the disease is not fatal and minor degrees of CD are accepted as "part of the job" but it may affect their health, work, and QoL adversely. ${ }^{5,6}$ Topical corticosteroids have been the mainstay in the treatment of eczema for more than three decades and are still the preferred agents in the symptomatic management of this clinical entity. ${ }^{7,8}$ Different complementary and alternative medicine (CAM) therapies, including homeopathy, have been reported to be of use in CD symptoms. ${ }^{9-11}$

The existing research evidence for homeopathy in atopic dermatitis remains inconclusive regarding the efficacy of the therapy; ${ }^{12-19} \mathrm{CD}$ has remained seriously under-researched. The homeopathic medicine Vinca minor (VM) is prepared from the plant VM Linn., also known as lesser periwinkle. It contains around 50 alkaloids of which the main alkaloid is Vincamine. In homeopathic literature, it has been mentioned for the treatment of $\mathrm{CD}^{20-27}$ However, to the authors' knowledge, no systematic research has yet been conducted using VM in CD.

In homeopathy, we use repertories showing which medicines are indicated when certain symptoms are present. But the meaning of the typefaces in the repertory is not very transparent. Kent used three grades in his repertory: ${ }^{20}$

1. Bold (3 marks)/1st grade: Found frequently in "all or the majority" of provers, confirmed by reproving, and verified clinically on the sick;

2. Italics (2 marks)/2nd grade: Symptoms found in few provers, confirmed by reproving, but occasionally verified clinically on the sick; and
3. Plain Roman (1 mark)/3rd grade: Symptoms experienced "now and then" in proving, not yet confirmed by reproving but verified by curing patients-so accepted as clinical symptom only.

Hitherto repertory entries have largely been based on single observations of a symptom occurring in a proving or in a "cured" case: that is, the absolute occurrence of symptoms, and eventually, that too, based on expert opinion, usually of very few experts. Moreover, their experience is seldom systematically collected and mostly memory-based. Even experienced homeopathic physicians have a limited number of successful cases concerning one medicine. This is a systematic and serious bias. This system of absolute grading of medicines in rubrics is fundamentally problematic. These are not relative statements; so, the more a medicine is prescribed, the more frequently corresponding symptoms will be confirmed and verified. Thus, the frequently used "polychrests" become over-represented in repertory rubrics. Besides, the size of repertory rubrics varies much more than expected from the prevalence of symptoms. Large rubrics tend to have superfluous entries, whereas in small rubrics medicines are missing. ${ }^{28}$ For example, the 2013 version of the Complete Repertory lists 19,210 entries (including main rubrics and sub-rubrics) for the homeopathic medicine Lycopodium clavatum (Lyc), while Kent's repertory accounts for "only" 6,805..$^{29}$ This is due to mere chance. If a medicine is prescribed frequently, sooner or later every symptom will turn up in a patient responding well to this medicine, more so in common symptoms (represented by large symptom rubrics). Another point is that the same homeopathic repertory has been used all over the world for more than a century despite huge climatological, cultural, and other variations and historical developments. Homeopathic practitioners in cold and in warm countries use the same repertory rubrics for being warm-blooded or cold-blooded and for influences of weather. Cultural differences do not seem to hamper the use of rubrics concerning emotions and food desires; we have no different rubrics for different countries or cultures. Apparently homeopathic practitioners use their clinical expertise to correct for such confounders, but it is uncertain how much bias these confounders could cause. ${ }^{30}$

Repertory entries should be replaced by relative grading: that is, a symptom is an indication for a medicine when it occurs more frequently in individuals cured by the medicine than in the rest of the population. This is intuitively understandable, but also based on Bayes' theorem. Thomas Bayes, 
the 18th century English clergyman and mathematician, described "conditional probabilities" in terms of beliefs and degrees of uncertainty: ${ }^{31}$

- Posterior odds $=$ Likelihood ratio $($ LR $) \times$ prior odds

- Odds = Chance/(1 - Chance $)$

- $\mathrm{LR}=$ prevalence of symptom in target population/prevalence of symptom in remainder of the population.

- Target population $=$ population responding well to the medicine.

- LR can be denoted as $\operatorname{LR}(+)$ if the symptom is present, or $\operatorname{LR}(-)$ if the symptom is absent.

The practical implication of Bayes' theorem is that if LR $(+)>1$, the odds/chance increases that the medicine will work. This odds/chance increases more if LR is higher: that is, if the difference between target population and remainder of the (practice) population is larger. The opposite is true for LR $(-)<1$ : odds/chance decreases if the symptom is absent; more so if LR is lower. Thus, LR provides a better indication for the prognostic value of a homeopathic symptom than the present repertory entries. How LR can be translated into repertory entries (LR values or typography) is still being researched and depends on the actual values that will be found in assessments of a considerable number of symptoms. $^{32}$

Thus, LR may yield a better estimation of a medicine's indication than the existing method of entry of symptoms into materia medica and repertories. So, the repertory entries must be based on the prevalence of the symptom in the medicine population compared with the prevalence in the remainder of the population. This prevalence can only be assessed by systematic scientific research, and preferably prospectively. ${ }^{33,34}$ This involves checking of symptoms in every new patient and taking the symptoms out of their context. Bayesian theory shows that the prevalence of the symptom is the only correct criterion. A symptom is an indication for a specific medicine only if the prevalence of that symptom is higher in the population cured by that medicine than in the rest of the population. This Bayesian principle has been recommended to be applied radically to all branches of medicine, not just to CAM. ${ }^{35}$

We estimated LRs of the four symptoms of CD of VM, selected after a literature search of materia medica: ${ }^{21-27}$

1. Great sensitiveness of skin, with redness and soreness from slightest rubbing;

2. Weeping eczema with foul, thick crusts;

3. Itching amelioration in open air; and

4. CD of scalp.

\section{Methods}

\section{Study Design}

This prospective, observational, patient outcome study was conducted at five different practice settings over 18 months. The study settings were in two states of India-Chhattisgarh and West Bengal-and included two semi-urban homeopathy institutions in Chhattisgarh, and one rural practice, one homeopathic hospital, and one homeopathy private practice in West Bengal.

The study protocol (unpublished) was submitted as $\mathrm{PhD}$ synopsis of the corresponding author to the Homoeopathy University, Jaipur, Rajasthan, India, and was approved by the Institutional Ethics Committee (HU/2016/1240/A).

\section{Participants}

Inclusion criteria were patients suffering from CD for 1 year or longer, age 18 to 65 years, both sexes, ability to read Bengali and providing written consent to participate. Exclusion criteria were unwillingness to participate or to comply with the study requirements, too unwell to take part, declined to provide consent, other uncontrolled pulmonary or systemic diseases and/or pathologies affecting QoL, psychiatric illness, pregnancy or lactation, ongoing use of homeopathic remedies for any chronic disease, self-reported immune-compromised state, and drug or substance abuse and/or dependence.

\section{Intervention}

All eligible patients suffering from $C D$ symptoms were first matched prospectively with any of the four CD symptoms of VM. If similarities were detected, they were prescribed with VM in centesimal potencies as per homeopathic principles. Otherwise, the patients were prescribed with other medicines as per indications. In homeopathic centesimal potencies (6C, 30C, 200C, 1,000C, and 10,000C), each dose consisted of four cane sugar globules medicated with the indicated medicine, preserved in $90 \% \mathrm{v} / \mathrm{v}$ ethanol. Repetition was dependent on the individual requirement of the case. Each dose was instructed to be taken orally on clean tongue and with empty stomach. Duration of therapy was 6 months. Single individualized medicine was prescribed on each occasion, taking into account presenting symptom totality, constitutional features, and miasmatic expressions. Dose was also individualized and was based on physicians' judgment of susceptibility. Subsequent prescriptions were generated as per Kent's observations and Hering's law. Each of the prescribers in the study possessed at least bachelor's degree in homeopathy, with more than 5 years of experience of practicing classical homeopathy. Results of treatment were evaluated at follow-up consultations after 3 and 6 months. Changes were assessed by the tools described in outcomes.

\section{Outcomes}

The following outcomes were regarded as indicating effects of the prescribed medicines:

1. Glasgow Homeopathic Hospital Outcome Scale (GHHOS) scale, assessed after 3 and 6 months. In a few preliminary validation studies, the outcomes obtained using GHHOS were found to be significantly correlated with the European QOL transition scale, the Measure Yourself Medical Outcome Profile, and the Patient Enablement Instrument, suggesting it as a valid and sensitive tool for measuring change in relation to impact on daily living. ${ }^{36,37}$ The 
GHHOS score describes improvement or deterioration; but GHHOS $\geq+2$ is considered an indication of a homeopathic effect, because of improvement in more than just the presented complaint.

2. Scoring Atopic Dermatitis (SCORAD), assessed at baseline, after 3 and 6 months; end point 6 months. It is the most widely used disease-severity scale in $\mathrm{AD}$ but it is also used in CD. ${ }^{38}$ It was developed in 1993 by the European Task Force on Atopic Dermatitis. ${ }^{39}$ The SCORAD index uses the rule of nines to assess disease extent and evaluates five clinical characteristics to determine disease severity: (1) erythema, (2) edema/papulation, (3) oozing/crusts, (4) excoriation, and (5) lichenification. SCORAD also assesses subjective symptoms of pruritus and sleep loss with Visual Analog Scale. These three aspects-extent of disease, disease severity, and subjective symptoms-combine to give a maximum possible score of 103. Although it is a combined score, the three aspects can be separated and used individually if necessary. Of all the severity scales used in $A D$, it is the most widely validated disease-severity instrument. ${ }^{40}$ SCORAD has been found to be valid and reliable, and it has shown excellent agreement with global assessments of disease severity. ${ }^{41}$

3. Dermatology Life Quality Index (DLQI) questionnaire: assessed at baseline, after 3 and 6 months; end point 6 months. It is one of the most frequently used QoL instruments in dermatological conditions. ${ }^{41}$ It was developed in 1994 by Drs. Finlay and Khan to measure QoL in routine clinical practice in adults over the age of $18 .^{42}$ DLQI is a 10 -item questionnaire that enquires about skin symptoms, feelings of embarrassment, and how skin disease has affected day-to-day activities, working and social life. Each question on DLQI is scored from 0 to 3, with a maximum score of 30 and high scores representing worse QoL. In the original article by Finlay and Khan, patients with AD had the worst QoL as measured by DLQI compared with the other skin diseases assessed in the study. DLQI has been extensively validated in multiple studies. ${ }^{43,44}$ A 10-year review of the literature found that DLQI is highly specific for assessing decrements in QoL in patients with AD compared with the general population. It also has high repeatability, internal consistency, and sensitivity to change. ${ }^{44}$ In the current study, the authors used the translated and pre-validated Bengali version of the DLQI questionnaire. ${ }^{45}$

\section{Statistical Methods}

All the collected data in the standardized format were recorded in a specially designed Excel spreadsheet, where they underwent statistical analysis-both descriptive and inferential. The intention-to-treat sample was analyzed. Missing values were calculated using the last-value-carried-forward method. Descriptive statistics were presented in terms of absolute values, percentages, means, and standard deviations, as appropriate. LRs of the four symptoms were estimated as per the patient-rated outcomes ( +4 to -4 ; +1 to +4 : improved; -1 to -4 : deteriorated; 0 : no change) obtained on GHHOS measures ${ }^{28,34}$ from the sample of 390 $\mathrm{CD}$ patients after formation of a-d cells in the $2 \times 2$ contingency tables as follows:

1. Number of patients with a certain symptom improved by VM;

2. "Remainder of the study sample" presenting with the symptom;

3. Number of patients with absence of the symptom, still improved by the medicine under question; and

4. "Remainder of the study sample" not having the symptom.

Parametric tests were used as inferential statistics accordingly. Dependent observations of continuous outcomes at baseline and at different points of time were compared using paired $t$-test. For continuous data, changes in outcomes obtained longitudinally at different points of time were tested using post hoc repeated measure analysis of variance (ANOVA). Correlations between GHHOS and changes in SCORAD, and between GHHOS and changes in DLQI, were assessed using scatter plots to check how significantly the changes obtained using a homeopathy-specific outcome measure (i.e., GHHOS) correlated with an AD-related symptom severity-specific outcome (i.e., SCORAD) and any dermatological condition-specific QoL measuring outcome (i.e., DLQI). $p$-Values were set at less than 0.05 (two-tailed) as statistically significant and were computed using chisquared test from $2 \times 2$ contingency tables with Yates' continuity correction. SPSS-IBM version 20 software for Windows was used for analysis of data.

\section{Results}

\section{Participant Flow}

In the VM sample $(n=74)$, the numbers of drop-outs were 4 and 7 after 3 and 6 months, respectively. A total of 512 patients suffering from $C D$ were screened initially; 57 were screened out. The resulting 455 patients underwent detailed screening as per specified eligibility criteria; 65 were excluded for varying reasons. A total of 390 patients were enrolled in the study. Of this total, 74 were prescribed VM as per individualization, and the remaining 316 were prescribed other medicines (-Fig. 1).

\section{Recruitment}

The study started in June 2016 and ended in December 2017.

\section{Baseline Data}

The majority of the patients were female (64.9\%) and spanned 18 to 35 years of age (43.2\%). Details of baseline socio-demographic characteristics in terms of age, gender, residence, education, occupation, monthly family income, and marital status have been presented in -Table 1.

\section{Numbers Analyzed}

After 6 months, 67 individuals were protocol-compliant in the VM sample. Fifty were responders: that is, improved according to all the three outcome measures. 


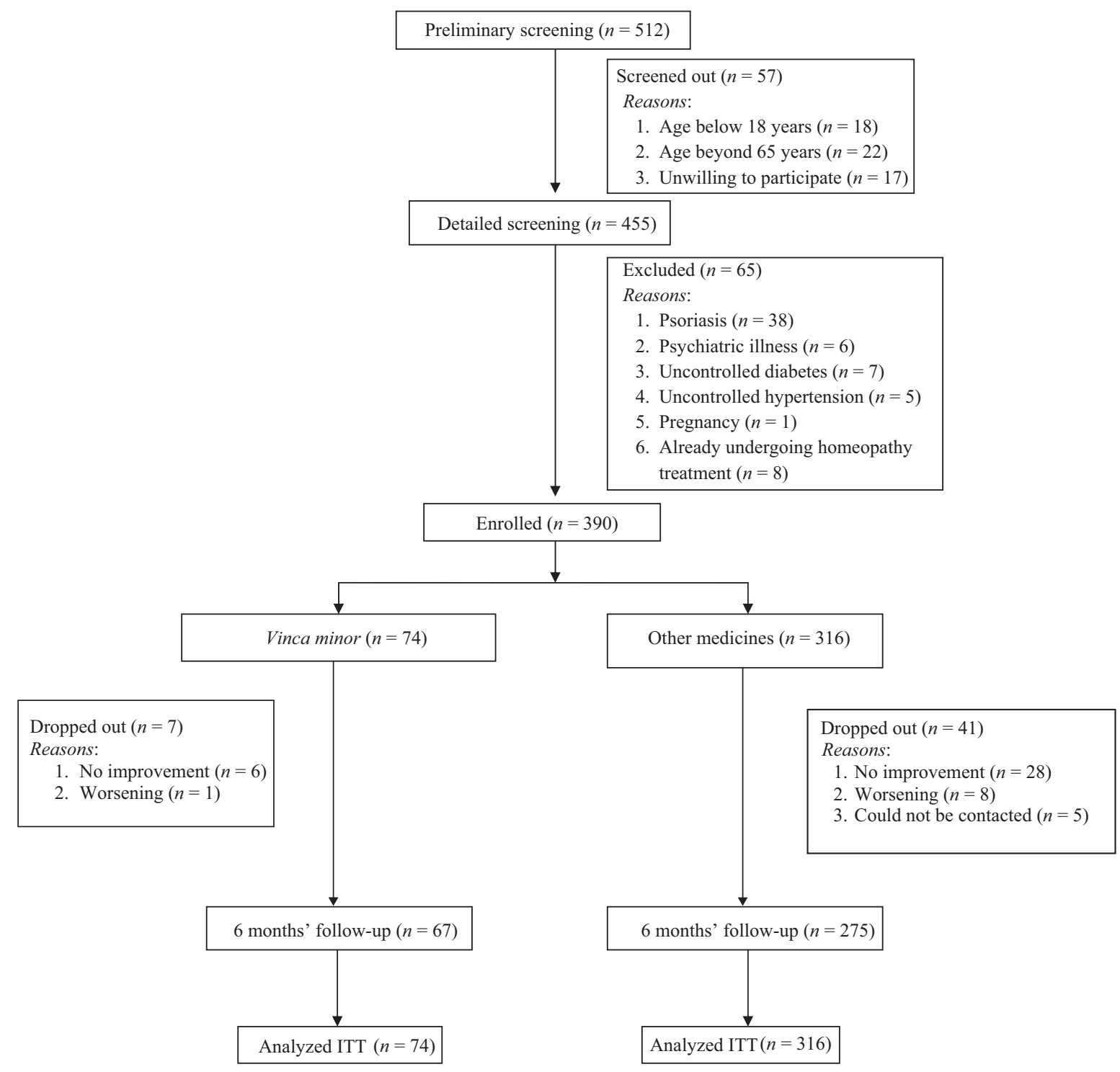

Fig. 1 Participant flow diagram. Abbreviation: ITT, intention-to-treat.

\section{Changes in Outcome Measures}

- Estimation of $\operatorname{LR}(+)$ and $\operatorname{LR}(-)$ : The calculated LRs of the four selected symptoms were as follows:

Symptom 1: Great sensitiveness of skin, with redness and soreness from slightest rubbing: LR (+) 1.29 (95\% CI: 0.652.60) and LR (-) 0.96 (95\% CI: 0.84-1.09), $p=0.622$.

Symptom 2: Weeping eczema with foul, thick crusts: LR (+) 1.48 (95\% CI: 0.80-2.74) and LR (-) 0.93 (95\% CI: 0.801.07), $p=0.316$.

Symptom 3: Itching amelioration in open air: LR $(+) 1.70$ (95\% CI: 0.94-3.07) and LR (-) 0.90 (95\% CI: 0.77-1.04), $p=0.133$.

Symptom 4: CD of scalp: LR (+) 1.36 (95\% CI: 0.74-2.51) and LR (-) 0.94 (95\% CI: 0.81-1.09), $p=0.448$.

Thus, among the four symptoms, only symptom 3 had LR value $>1.5$ (-Table 2 ).

- SCORAD changes: Over 3 and 6 months of intervention, SCORAD values decreased significantly from $61.0 \pm 12.7$ to
$55.2 \pm 11.8$ and $47.5 \pm 17.1$, respectively. These reductions were statistically significant.

- DLQI changes: Over 3 and 6 months of intervention, DLQI values were also reduced significantly from $14.9 \pm 4.7$ to $13.1 \pm 4.0$ and $11.1 \pm 4.7$, respectively. These reductions were also statistically significant.

Mean changes in SCORAD and DLQI readings over 3 and 6 months have been presented in - Table 3.

- Correlation analysis: Scatter plots showed strong negative linear correlations between GHHOS and SCORAD changes, and GHHOS and DLQI changes after 3 and 6 months (-Figs. 2-5).

\section{Medicines Used}

Apart from VM $(n=74), 19$ different other remedies were used for the rest of the sample (i.e., $n=316$ ). Among other medicines, Graphites, Petroleum, Fagopyrum aesculentum, Rhus toxicodendron, Sulfur, Arsenicum album, Dulcamara, 
Table 1 Socio-demographics of the Vinca minor study sample $(n=74)$

\begin{tabular}{|c|c|}
\hline Features & Study sample \\
\hline Age (years) ${ }^{\mathrm{a}}$ & $40.2 \pm 15.2$ \\
\hline \multicolumn{2}{|l|}{ Age group (years) ${ }^{b}$} \\
\hline $18-35$ & $32(43.2)$ \\
\hline $36-50$ & $21(28.4)$ \\
\hline Above 50 & $21(28.4)$ \\
\hline \multicolumn{2}{|l|}{ Gender $^{\mathrm{b}}$} \\
\hline Male & $26(35.1)$ \\
\hline Female & $48(64.9)$ \\
\hline \multicolumn{2}{|l|}{ Residence $^{\mathrm{b}}$} \\
\hline Rural & $25(33.8)$ \\
\hline Semi-urban & $21(28.4)$ \\
\hline Urban & $28(37.8)$ \\
\hline \multicolumn{2}{|l|}{ Education $^{\mathrm{b}}$} \\
\hline 10th std. or less & $26(35.1)$ \\
\hline 12th std. & $27(36.5)$ \\
\hline Graduate or above & $21(28.4)$ \\
\hline \multicolumn{2}{|l|}{ Occupation $^{\mathrm{b}}$} \\
\hline Student & 11 (14.9) \\
\hline Dependent & $24(32.4)$ \\
\hline Self-employed & $26(35.1)$ \\
\hline Service & $13(17.6)$ \\
\hline \multicolumn{2}{|c|}{ Monthly family income ${ }^{\mathrm{b}}$} \\
\hline Less than 10,000 & $47(63.5)$ \\
\hline $10,000-30,000$ & $22(29.7)$ \\
\hline More than 30,000 & $5(6.8)$ \\
\hline \multicolumn{2}{|l|}{ Marital status ${ }^{\mathrm{b}}$} \\
\hline Married & $23(31.1)$ \\
\hline Single & $47(63.5)$ \\
\hline Others & $4(5.4)$ \\
\hline
\end{tabular}

${ }^{a}$ Continuous data presented as mean \pm standard deviation.

${ }^{\mathrm{b}}$ Categorical data presented as $n(\%)$
Kali arsenicosum, Antim ars, and Bovista were most frequently prescribed. These have been listed in decreasing order of frequency in - Table 4.

\section{Discussion}

We conducted an observational, prospective, patient-outcome study to assess LRs of four CD symptoms of the homeopathic medicine VM in six different practice settings on 390 patients (74 VM patients; 316 non-VM patients) over 1.5 years using three outcome scales-GHHOS, SCORAD, and DLQI, measured over 3 and 6 months. LRs of four symptoms were estimated: (1) great sensitiveness of skin, with redness and soreness from slightest rubbing $[\operatorname{LR}(+)$ 1.29]; (2) weeping eczema with foul, thick crusts $[\mathrm{LR}(+) 1.48]$; (3) itching amelioration in open air $[\operatorname{LR}(+)$ 1.70]; and (4) CD of scalp $[\operatorname{LR}(+)$ 1.36]. There were statistically significant reductions in SCORAD and DLQI scores also over 3 and 6 months. Thus, "itching amelioration in open air," with a relatively high yet statistically non-significant LR $(+)$, appears as the only one of our four investigated symptoms of VM that is worth considering as having prognostic value. ${ }^{32}$

We selected four rather common symptoms of the disease as available in literature, and doing so and restricting the control group to patients with the same condition, it was expected to find the symptoms frequently both in patients treated successfully with VM and in the remainder of the patients. The obvious consequence was to get lower and nonsignificant LRs, as indeed we obtained. Also, the assessments of disease severity consistently showed substantial improvements, although the disease was long-standing. This may be partly explained by placebo effect, regression to the mean effects, and/or effects of other confounders that our study was not designed to control. We also cannot rule out overestimation of the treatment effect and undisclosed use of concurrent therapeutic modalities, if any, as inherent limitations of observational trial design.

Correlations between the general homeopathic GHHOS outcome measure and the two disease-specific outcome measures increased from 3 months to 6 months. These observations indicate that it had taken more time to reveal positive responses on GHHOS than on SCORAD or DLQI, from

Table 2 Likelihood ratios of the four symptoms of Vinca minor (VM) (as per GHHOS) $(n=390)$

\begin{tabular}{|c|c|c|c|c|c|c|c|c|c|c|}
\hline Symptoms & a & b & c & d & $\mathrm{LR}(+)$ & $95 \% \mathrm{Cl}$ & $\operatorname{LR}(-)$ & $95 \% \mathrm{Cl}$ & $\begin{array}{l}\mathrm{x}^{2} \text { value } \\
\text { (Yates) }\end{array}$ & $p$-Value \\
\hline $\begin{array}{l}\text { Great sensitiveness of skin, with } \\
\text { redness and soreness from } \\
\text { slightest rubbing }\end{array}$ & 8 & 42 & 42 & 298 & 1.29 & $0.65-2.60$ & 0.96 & $0.84-1.09$ & 0.24 & 0.622 \\
\hline $\begin{array}{l}\text { Weeping eczema with foul, thick } \\
\text { crusts }\end{array}$ & 10 & 46 & 40 & 294 & 1.48 & $0.80-2.74$ & 0.93 & $0.80-1.07$ & 1.00 & 0.316 \\
\hline Itching amelioration in open air & 11 & 44 & 39 & 296 & 1.70 & $0.94-3.07$ & 0.90 & $0.77-1.04$ & 2.25 & 0.133 \\
\hline CD of scalp & 10 & 50 & 40 & 290 & 1.36 & $0.74-2.51$ & 0.94 & $0.81-1.09$ & 0.58 & 0.448 \\
\hline
\end{tabular}

Abbreviations: $\mathrm{Cl}$, confidence interval; CD, contact dermatitis; GHHOS, Glasgow Homeopathic Hospital Outcome Scale; LR, likelihood ratio.

"a": The number of patients with the symptom improved by VM.

"b": Remainder of the study sample presenting with the symptom.

"c": Number of patients with absence of the symptom, still improved by the medicine.

"d": Remainder of the study sample not having the symptom. 
Table 3 Changes in observed outcomes over time in Vinca minor sample $(n=74)$

\begin{tabular}{|l|l|l|l|l|l|l|}
\hline Outcomes & Baseline & $\begin{array}{l}\text { After 3 } \\
\text { months }\end{array}$ & $\begin{array}{l}\text { Change } \\
(\mathbf{0 - 3} \text { months): } \\
\text { mean } \pm \text { SD }\end{array}$ & $\begin{array}{l}\text { After 6 } \\
\text { months }\end{array}$ & $\begin{array}{l}\text { Change (3-6 months): } \\
\text { mean } \pm \text { SD }\end{array}$ & $\begin{array}{l}\text { Change } \\
\text { (0-6 months): } \\
\text { mean } \pm \text { SD }\end{array}$ \\
\hline SCORAD & $61.0 \pm 12.7$ & $55.2 \pm 11.8$ & $5.8 \pm 6.2^{\mathrm{a}}$ & $47.5 \pm 17.1$ & $7.7 \pm 9.6^{\mathrm{b}}$ & $13.5 \pm 14.3^{\mathrm{c}, \mathrm{d}}$ \\
\hline DLQI & $14.9 \pm 4.7$ & $13.1 \pm 4.0$ & $1.7 \pm 2.3^{\mathrm{a}}$ & $11.1 \pm 4.7$ & $2.0 \pm 3.2^{\mathrm{b}}$ & $3.7 \pm 4.8^{\mathrm{c}, \mathrm{d}}$ \\
\hline
\end{tabular}

Abbreviations: ANOVA, analysis of variance; DLQI, Dermatology Life Quality Index; SCORAD, Scoring Atopic Dermatitis; SD, standard deviation. SCORAD: ${ }^{\mathrm{a}} p<0.001 ;{ }^{\mathrm{b}} p<0.001 ;{ }^{\mathrm{c}} p<0.001$; ${ }^{\mathrm{d}}$ One-way repeated measure ANOVA, Wilks's lambda $=0.509, F(2,72)=34.781, p<0.001$, $\eta=0.491$, that is, significant time effect (Lambda is the measure of the \% variance in dependent variables not explained by differences in levels of the independent variable. The null hypothesis is rejected when Wilks's lambda is close to zero. Eta $[\eta]$ is the square root of eta squared [ $\left.\eta^{2}\right]$, a measure of effect size for use in ANOVA and analogous to $R^{2}$ from multiple linear regression).

DLQI: ${ }^{\mathrm{a}} p<0.001{ }^{\mathrm{b}} p<0.001 ;{ }^{\mathrm{c}} p<0.001$; $^{\mathrm{d}}$ One-way repeated measure ANOVA, Wilks's lambda $=0.607, F(2,72)=23.310, p<0.001, \eta=0.393$, that is, significant time effect.

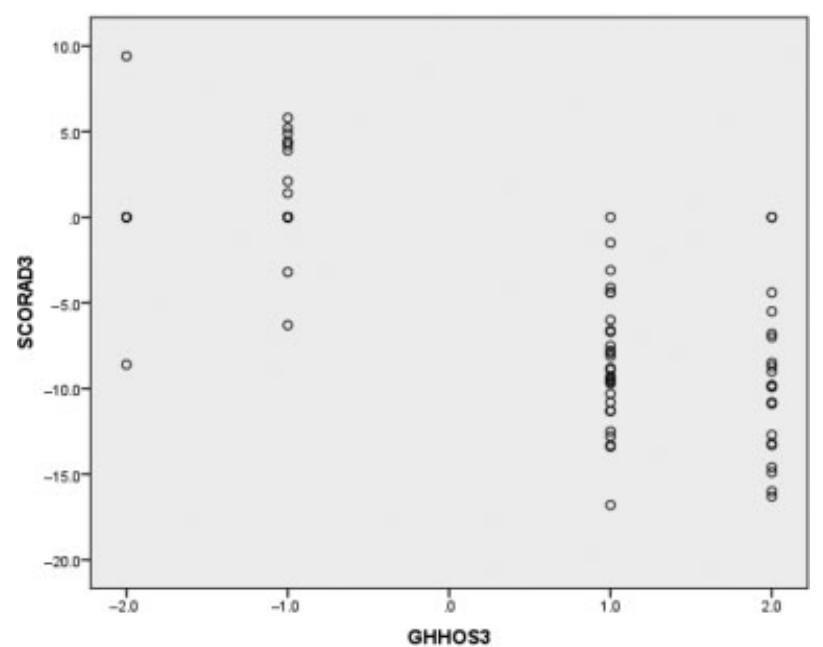

Fig. 2 Scatter plot showing correlations between GHHOS and SCORAD change over 3 months for VM sample $(n=74) ; r=-0.723$ (strong negative linear relationship); $p<0.001^{*}$.

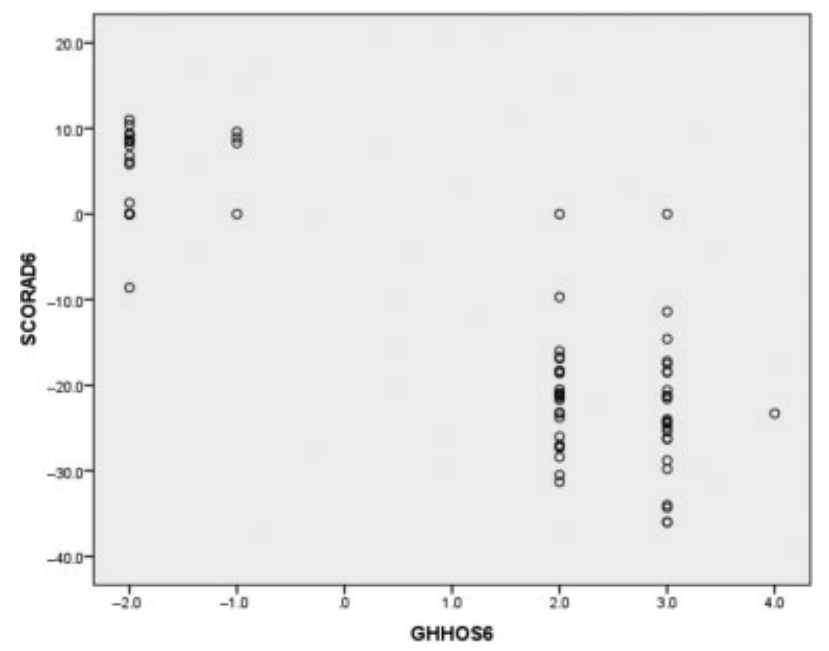

Fig. 3 Scatter plot showing correlations between GHHOS and SCORAD change over 6 months for VM sample $(n=74) ; r=-0.869$ (strong negative linear relationship); $p<0.001^{*}$.

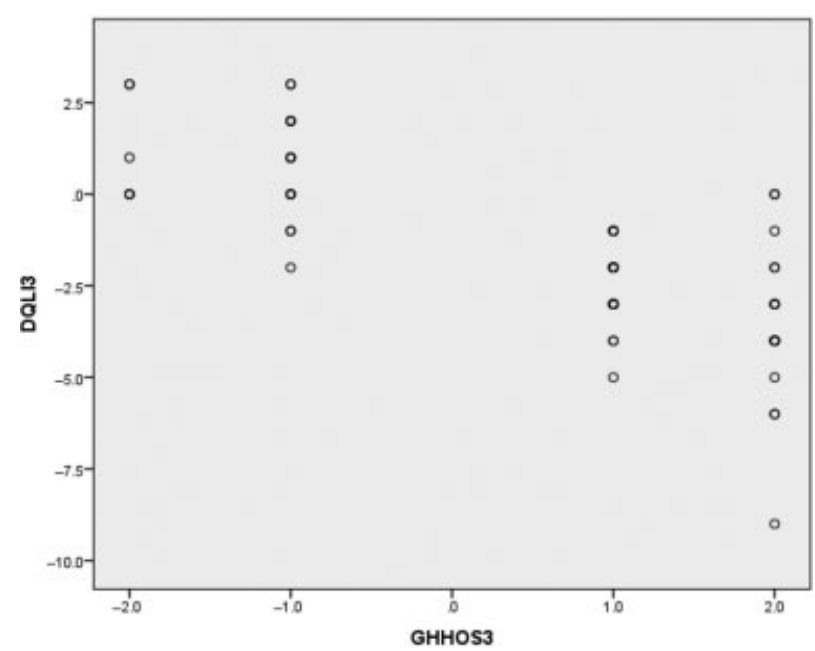

Fig. 4 Scatter plot showing correlations between GHHOS and DLQI change over 3 months for VM sample $(n=74) ; r=-0.776$ (strong negative linear relationship); $p<0.001^{*}$.

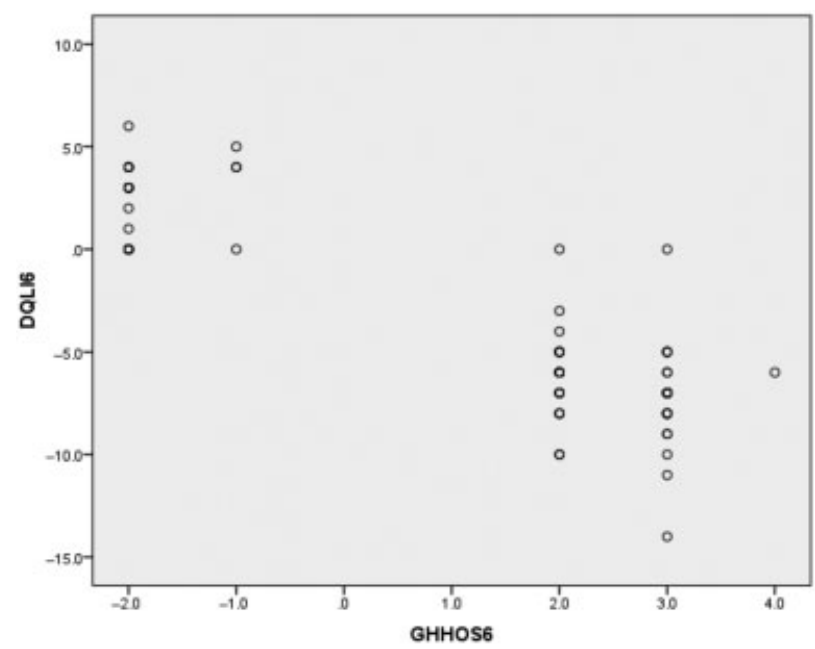

Fig. 5 Scatter plot showing correlations between GHHOS and DLQI change over 6 months for VM sample $(n=74) ; r=-0.875$ (strong negative linear relationship); $p<0.001^{*}$. 
Table 4 Other indicated medicines apart from Vinca minor $(n=316)$

\begin{tabular}{|l|l|l|}
\hline Medicines & Frequency & Percentage \\
\hline Graphites & 68 & 21.5 \\
\hline Petroleum & 51 & 16.1 \\
\hline Fagopyrum aesculentum & 30 & 9.5 \\
\hline Rhus toxicodendron & 23 & 7.3 \\
\hline Sulfur & 21 & 6.6 \\
\hline Arsenicum album & 17 & 5.4 \\
\hline Dulcamara & 15 & 4.7 \\
\hline Kali arsenicosum & 11 & 3.5 \\
\hline Antimonium arsenicosum & 10 & 3.2 \\
\hline Bovista & 10 & 3.2 \\
\hline Antimonium crudum & 8 & 2.5 \\
\hline Sepia succus & 8 & 2.5 \\
\hline Mercurius solubilis & 8 & 2.5 \\
\hline Psorinum & 7 & 2.2 \\
\hline Anagallis & 7 & 2.2 \\
\hline Rhus venenata & 7 & 2.2 \\
\hline Acid chrysorbinum & 5 & 1.6 \\
\hline Mezereum & 5 & 1.6 \\
\hline Skookum chuck & 5 & 1.6 \\
\hline
\end{tabular}

which improvements were detected earlier. This should be investigated in future research. It is quite common in homeopathic practice to get positive responses in chronic cases after 3 months, ${ }^{34}$ but possibly the impact on daily living as measured by GHHOS accrues more slowly.

Following assessment of LRs from a cough population from the IIPCOS2 study, ${ }^{46}$ it was the very next prospective condition-confined assessment of LRs of symptoms. Instead of "whole population" (as used in LR calculation), a subpopulation of "patients suffering from a particular disease or condition" (i.e., CD) was considered. So, instead of seeking prevalence of symptoms in "whole population," prevalence of symptoms in CD sample was calculated. This prognostic factor research was performed regarding one condition, $C D$; hence, the outcome is valid for that condition only, and we assumed that the pre-selected medicines were effective for that condition. The reason behind the choice of this CD subpopulation was our pre-planned targeted research in this special condition. We also intended to develop a therapeutic guide for this disease condition by exploring utility of a relatively less-used medicine such as VM.

Prognostic factor research in sub-groups defined by conditions is only valid for these sub-groups. If a sub-group or condition is selected, we inherently also select a sub-group of medicines that are related to that condition more than others. Ideally, we should base our repertory entries on the relative occurrence of symptoms in populations that respond well to specific homeopathic medicines. This can be done by applying LR, and this is based on a comparison within the whole practice population. But there can be various reasons for restricting our research sample to subgroups of this whole population. However, we must be careful about complications. These complications are caused by restricting the group of assessed medicines, thus causing confirmation bias in selecting medicines. To elaborate, at first, there is overconfidence that a medicine can or cannot work if a symptom is present or absent and results are overestimated accordingly. Thus, the prevalence of the symptom in the sample that responds well to a particular medicine is overestimated in the beginning. This bias could have been eased further if we could have used longer followups. However, generalizability was increased by use of standardized or validated symptoms and outcome measures and the multi-center gathering of data.

\section{Conclusion}

There was insufficient evidence to attribute any of the four assessed symptoms clearly to the homeopathic medicine VM. Though non-significant, higher LR was observed for "itching amelioration in open air." Symptoms available in the homeopathic materia medica are perhaps misleading and probably over-represented and influenced by mere chance. Despite its limitations, our research shows how homeopathic symptoms can be assessed scientifically. The gap between expert opinions in homeopathy and valid dataat least for VM-has been narrowed with this investigation. Our study should be replicated by others to improve external validity and thus help resolve discrepancies between contemporary research data and the existing materia medica.

\section{Highlights}

- Four symptoms of CD of VM were selected for LR estimation: (1) great sensitiveness of skin, with redness and soreness from slightest rubbing; (2) weeping eczema with foul, thick crusts; (3) itching amelioration in open air; and (4) CD of scalp.

- Though non-significant, a high LR was observed only for the symptom "Itching amelioration in open air," and it appeared as the only probable symptom of VM as having prognostic value.

\section{Funding}

We received no funding for the project. The institution has no role to play in analysis of the study results and publication of the article.

\section{Conflict of Interest}

We declare no conflict of interest. The study was performed as $\mathrm{PhD}$ thesis of the corresponding author.

\section{Acknowledgement}

The authors sincerely thank the staff, pharmacist, and the patients for their sincere participation in the study. We also acknowledge Dr. Robert T. Mathie, Senior Deputy Editor, Homeopathy for his valuable comments and inputs to improve the article. 


\section{References}

1 Sasseville D. Occupational contact dermatitis. Allergy Asthma Clin Immunol 2008;4:59-65

2 Ale IS, Maibach HI. Irritant contact dermatitis. Rev Environ Health 2014;29:195-206

3 Lachapelle JM. Allergic contact dermatitis: clinical aspects. Rev Environ Health 2014;29:185-194

4 Alchorne AO, Alchorne MM, Silva MM. Occupational dermatosis. An Bras Dermatol 2010;85:137-145

5 Sarma N. Occupational allergic contact dermatitis among construction workers in India. Indian J Dermatol 2009;54:137-141

6 Anderson SE, Meade BJ. Potential health effects associated with dermal exposure to occupational chemicals. Environ Health Insights 2014;8:51-62

7 Cohen DE, Heidary N. Treatment of irritant and allergic contact dermatitis. Dermatol Ther (Heidelb) 2004;17:334-340

8 Holness DL. Occupational skin allergies: testing and treatment (the case of occupational allergic contact dermatitis). Curr Allergy Asthma Rep 2014;14:410

9 Jacobsson I, Jönsson AK, Gerdén B, Hägg S. Spontaneously reported adverse reactions in association with complementary and alternative medicine substances in Sweden. Pharmacoepidemiol Drug Saf 2009;18:1039-1047

10 Noiesen E, Munk MD, Larsen K, Høyen M, Agner T. Use of complementary and alternative treatment for allergic contact dermatitis. Br J Dermatol 2007;157:301-305

11 Bascom A. Complementary and alternative therapies in occupational health. Part II-Specific therapies. AAOHN J 2002; 50:468-477

12 Siebenwirth J, Lüdtke R, Remy W, Rakoski J, Borelli S, Ring J. Effectiveness of a classical homeopathic treatment in atopic eczema. A randomised placebo-controlled double-blind clinical trial [Article in German]. Forsch Komplement Med 2009;16:315-323

13 Keil T, Witt CM, Roll S, et al. Homoeopathic versus conventional treatment of children with eczema: a comparative cohort study. Complement Ther Med 2008;16:15-21

14 Witt CM, Brinkhaus B, Pach D, et al. Homoeopathic versus conventional therapy for atopic eczema in children: medical and economic results. Dermatology 2009;219:329-340

15 Witt CM, Lüdtke R, Willich SN. Homeopathic treatment of children with atopic eczema: a prospective observational study with two years follow-up. Acta Derm Venereol 2009;89:182-183

16 Itamura R, Hosoya R. Homeopathic treatment of Japanese patients with intractable atopic dermatitis. Homeopathy 2003;92:108-114

17 Rossi E, Bartoli P, Bianchi A, Da Frè M. Homeopathy in paediatric atopic diseases: long-term results in children with atopic dermatitis. Homeopathy 2012;101:13-20

18 Eizayaga JE, Eizayaga JI. Prospective observational study of 42 patients with atopic dermatitis treated with homeopathic medicines. Homeopathy 2012;101:21-27

19 Ernst E. Homeopathy for eczema: a systematic review of controlled clinical trials. Br J Dermatol 2012;166:1170-1172

20 Kent JT. Lectures on Homoeopathic Philosophy. Chapters 32, 33. New Delhi: B. Jain Publishers (P) Ltd.; 2007:203-209

21 Hering C. Guiding Symptoms of Our Materia Medica. New Delhi: B. Jain Publishers (P) Ltd.; 1974

22 Clarke JH. A Dictionary of Practical Materia Medica. New Delhi: B. Jain Publishers (P) Ltd.; 1984

23 Allen TF. The Encyclopaedia of Pure Materia Medica. New Delhi: B. Jain Publishers (P) Ltd.; 2000

24 Boericke W. New Manual of Homeopathic Materia Medica with Repertory: 3rd Revised \& Augmented Edition based on 9th edition. New Delhi: B. Jain Publishers (P) Ltd.; 2010

25 Choudhury NM. A Study on Materia Medica. New Delhi: B. Jain Publishers (P) Ltd.; 2009
26 Hempel CJ. A New and Comprehensive System of Materia Medica and Therapeutics: Arranged upon a Physiologico-pathological basis for the use of Practitioners and Students of Medicine. 3rd ed. New York: Radde; 1865

27 Hughes R, Dake JP. A Cyclopedia of Drug Pathogenesy. New Delhi: B. Jain Publishers; 1992

28 Rutten ALB, Stolper CF, Lugten RF, Barthels RW. Statistical analysis of six repertory rubrics after prospective assessment applying Bayes' theorem. Homeopathy 2009;98:26-34

29 Eizayaga JE, Pozzi MI, Canan MC, Saravia L. Prevalence and likelihood ratio of symptoms in patients with good therapeutic response to Lycopodium clavatum. A retrospective study. Homeopathy 2016;105:78-83

30 Rutten ALB, Muraleedharan KC, Shinde VH, Manchanda RK. What is a homoeopathic symptom, in daily practice and research? Indian J Res Homoeopathy. 2017;11:12-20

31 Bayes T. An essay toward solving a problem in the doctrine of chances. Philos Trans R Soc Lond 1763;53:370-418

32 Rutten ALB, Stolper CF, Lugten RFG, Barthels RWJM. Synchronising the Repertory with Research Data. Homeopathy 2008;97:16-21

33 Rutten ALB. Prognostic factor research in homoeopathy. Indian J Res Homoeopathy. 2016;10:59-65

34 Koley M, Saha S, Das KD, et al. Prospective evaluation of few homeopathic rubrics of Kent's repertory from Bayesian perspective. J Evid Based Complementary Altern Med 2016;21:277-281

35 Walach H, Roberti di Sarsina P, Tassinari M. Data about (complementary and alternative) medicine are irrelevant, because we are all Bayesians. Eur J Intern Med 2015;26:e10-e11

36 Bikker AP, Mercer SW, Reilly D. A pilot prospective study on the consultation and relational empathy, patient enablement, and health changes over 12 months in patients going to the Glasgow Homoeopathic Hospital. J Altern Complement Med 2005; 11:591-600

37 Reilly D, Mercer SW, Bikker AP, Harrison T. Outcome related to impact on daily living: preliminary validation of the ORIDL instrument. BMC Health Serv Res 2007;7:139

38 Maiti R, Sirka CS, Shaju N, Hota D. Halometasone monohydrate $(0.05 \%)$ in occupational contact dermatitis. Indian J Pharmacol 2016;48:128-133

39 Kunz B, Oranje AP, Labrèze L, Stalder JF, Ring J, Taïeb A. Clinical validation and guidelines for the SCORAD index: consensus report of the European Task Force on Atopic Dermatitis. Dermatology 1997;195:10-19

40 Charman C, Williams H. Outcome measures of disease severity in atopic eczema. Arch Dermatol 2000;136:763-769

41 Rehal B, Armstrong AW. Health outcome measures in atopic dermatitis: a systematic review of trends in disease severity and quality-of-life instruments 1985-2010. PLoS One 2011;6: e17520

42 Finlay AY, Khan GK. Dermatology Life Quality Index (DLQI)-a simple practical measure for routine clinical use. Clin Exp Dermatol 1994;19:210-216

43 Badia X, Mascaró JM, Lozano R; The Cavide Research Group. Measuring health-related quality of life in patients with mild to moderate eczema and psoriasis: clinical validity, reliability and sensitivity to change of the DLQI. Br J Dermatol 1999;141: 698-702

44 Lewis V, Finlay AY. 10 years experience of the Dermatology Life Quality Index (DLQI). J Investig Dermatol Symp Proc 2004; 9:169-180

45 Available at. http://sites.cardiff.ac.uk/dermatology/files/2014/04/ DLQI_Bengali.pdf. Accessed March 12, 2015

46 Miglani A, Rutten L, Manchanda RK. Generalisability of prognostic factor research: further analysis of data from the IIPCOS2 study. Homeopathy 2017;106:155-159 